\title{
Radiative Decay Widths of Ground and Excited States of Vector Charmonium and Bottomonium
}

\author{
Hluf Negash $^{1}$ and Shashank Bhatnagar ${ }^{2}$ \\ ${ }^{1}$ Department of Physics, Samara University, P.O. Box 132, Samara, Ethiopia \\ ${ }^{2}$ Department of Physics, University Institute of Sciences, Chandigarh University, Mohali 140413, India
}

Correspondence should be addressed to Hluf Negash; h2002hluf@yahoo.com

Received 26 January 2017; Accepted 14 May 2017; Published 20 June 2017

Academic Editor: Thierry Grandou

Copyright ( 2017 Hluf Negash and Shashank Bhatnagar. This is an open access article distributed under the Creative Commons Attribution License, which permits unrestricted use, distribution, and reproduction in any medium, provided the original work is properly cited. The publication of this article was funded by SCOAP ${ }^{3}$.

\begin{abstract}
We study the radiative decay widths of vector quarkonia for the process of $J / \psi(n S) \rightarrow \eta_{c}(n S) \gamma$ and $\Upsilon(n S) \rightarrow \eta_{b}(n S) \gamma($ for principal quantum numbers $n=1,2,3$ ) in the framework of Bethe-Salpeter equation under the covariant instantaneous ansatz using a $4 \times 4$ form of BSE. The parameters of the framework were determined by a fit to the mass spectrum of ground states of pseudoscalar and vector quarkonia, such as $\eta_{c}, \eta_{b}, J / \psi$, and $\Upsilon$. These input parameters so fixed were found to give good agreements with data on mass spectra of ground and excited states of pseudoscalar and vector quarkonia, leptonic decay constants of pseudoscalar and vector quarkonia, two-photon decays, and two-gluon decays of pseudoscalar quarkonia in our recent paper. With these input parameters so fixed, the radiative decay widths of ground $(1 S)$ and excited $(2 S, 3 S)$ states of heavy vector quarkonia $(J / \Psi$ and $\Upsilon)$ are calculated and found to be in reasonable agreement with data.
\end{abstract}

\section{Introduction}

Studies on mass spectra and decays of heavy quarkonia $(c \bar{c}$ and $b \bar{b}$ ) have become a hot topic in recent years, due to observation of many new states at various high energy accelerators at BABAR, Belle, CLEO, and BES-III collaborations [1-4]. All this has opened up new challenges in theoretical understanding of heavy hadrons and provide an important tool for exploring the structure of these simplest bound states in QCD and for studying the nonperturbative (long distance) behavior of strong interactions.

As regards the dynamical framework, to investigate these properties is concerned; many nonperturbative approaches, such as lattice QCD [5], chiral perturbation theory [6], QCD sum rules [7], heavy-quark effective theory [8], NRQCD [9], dynamical-equation based approaches like SchwingerDyson equation and Bethe-Salpeter equation (BSE) [10-15], and potential models [16] have been proposed to deal with the long distance property of QCD.

Bethe-Salpeter equation (BSE) $[11,15,17-19]$ is a conventional approach in dealing with relativistic bound state problems. From the solutions, we can obtain useful information about the inner structure of hadrons, which is also crucial in treating hadronic decays. The BSE framework which is firmly rooted in field theory provides a realistic description for analyzing hadrons as composite objects. Despite its drawback of having to input model-dependent kernel, these studies have become an interesting topic in recent years, since calculations have shown that BSE framework using phenomenological potentials can give satisfactory results as more and more data are being accumulated. The BSE is frequently adopted as starting point of QCD inspired models, due to the fact that this equation has a firm base in quantum field theory.

In a recent work [20,21], we employed a $4 \times 4$ representation for two-body $(q \bar{q})$ BS amplitude for calculating both the mass spectra and the transition amplitudes for various processes. However, the price one pays in this approach is to solve a coupled set of Salpeter equations for both pseudoscalar and vector quarkonia. However, in [21], we explicitly showed that these coupled Salpeter equations can indeed get decoupled in the heavy-quark approximation, leading to mass spectral equations with analytical solutions for both masses, as well as eigenfunctions for all the ground and excited states of pseudoscalar and vector $c \bar{c}$ and $b \bar{b}$ systems 
in an approximate harmonic oscillator basis. These analytical forms of eigenfunctions for ground and excited states so obtained were used to evaluate the transition amplitudes for different processes in [21]. Thus, in [21], we had calculated the mass spectrum, weak decay constants, two-photon decay widths and two-gluon decay widths of ground (1S) and radially excited $(2 S, 3 S, \ldots)$ states of pseudoscalar charmonium and bottomonium such as $\eta_{c}$ and $\eta_{b}$, as well as the mass spectrum and leptonic decay constants of ground state $(1 S)$ and excited $(2 S, 1 D, 3 S, 2 D, 4 S$, and $3 D)$ states of vector charmonium and bottomonium such as $J / \psi$ and $\Upsilon$, using this formulation of $4 \times 4$ Bethe-Salpeter equation under covariant instantaneous ansatz (CIA). Our results were in good agreement with data (wherever available) and other models. However, in all the above processes, the quark antiquark loop involved a single hadron-quark vertex, which was simple to handle.

However for the transitions such as $V \rightarrow P+\gamma$, the process requires calculation of triangle quark-loop diagram involving two hadron-quark vertices and is difficult to evaluate in BSECIA, which gives rise to complexities in amplitudes. However in $[22,23]$, they demonstrated an explicit mathematical procedure for handling such problems in $4 \times 4$ representation of BSE. Thus, in the present work, we will precisely apply an instantaneous formalism employing $4 \times 4$ BSE under CIA for transitions involving the process, $V \rightarrow P \gamma$, where such problems do not enter in the calculations of [21].

This paper is organized as follows. In Section 2, we give the formulation of $4 \times 4$ BSE under CIA. In Section 3, we give the derivation of the hadronic process $V \rightarrow P+\gamma$ in the framework of $4 \times 4$ BSE under CIA and calculate its radiative decay widths. The numerical results for radiative decay widths of the processes are worked out. Section 4 is reserved for discussions and conclusion.

\section{Formulation of BSE under CIA}

We give a short derivation of Salpeter equations in this section, giving only the main steps. The $4 \mathrm{D}$ BSE for $q \bar{q}$ comprising equal mass fermionic quarks of momenta $p_{1,2}$ and masses $m_{1}=m_{2}(=m)$, respectively, is written in $4 \times 4$ representation as

$$
\begin{aligned}
& \left(\not p_{1}-m_{1}\right) \Psi(P, q)\left(\not p_{2}+m_{2}\right) \\
& =\frac{i}{(2 \pi)^{4}} \int d^{4} q^{\prime} K\left(q, q^{\prime}\right) \Psi\left(P, q^{\prime}\right),
\end{aligned}
$$

where the $4 \times 4$ BS wave function is sandwiched between the inverse propagators of the quark and the antiquark, whose individual momenta $p_{1,2}$ are related to the internal momentum $q$ and total momentum $P$ of hadron of mass $M$ as $p_{1,2 \mu}=(1 / 2) P_{\mu} \pm q_{\mu}$. We further decompose the internal momentum, $q_{\mu}$, as the sum of its transverse component, $\widehat{q}_{\mu}=q_{\mu}-(q \cdot P) P_{\mu} / P^{2}$ (which is orthogonal to total hadron momentum $\left.P_{\mu}\right)$, and the longitudinal component, $\sigma P_{\mu}=(q$. P) $P_{\mu} / P^{2}$ (which is parallel to $P_{\mu}$ ). Thus, $q_{\mu}=(M \sigma, \widehat{q})$, where the transverse component, $\hat{q}$, is an effective $3 \mathrm{D}$ vector, while the longitudinal component, $M \sigma$, plays the role of the time component. The $4 \mathrm{D}$ volume element in this decomposition is $d^{4} q=d^{3} \hat{q} M d \sigma$. To obtain the 3D BSE and the hadronquark vertex, use an ansatz on the BS kernel $K$ in (1) which is assumed to depend on the $3 \mathrm{D}$ variables $\widehat{q}_{\mu}, \widehat{q}_{\mu}^{\prime}$ as

$$
K\left(q, q^{\prime}\right)=K\left(\widehat{q}, \hat{q}^{\prime}\right)
$$

Hence, the longitudinal component, $M \sigma$ of $q_{\mu}$, does not appear in the form $K\left(\widehat{q}, \widehat{q}^{\prime}\right)$ of the kernel and we define $3 \mathrm{D}$ wave function $\psi(\widehat{q})$ as

$$
\psi(\widehat{q})=\frac{i}{2 \pi} \int \operatorname{Md} \sigma \Psi(P, q) .
$$

Substituting (3) in (1), with definition of kernel in (2), we get a covariant version of Salpeter equation

$$
\begin{aligned}
& \left(\not p_{1}-m_{1}\right) \Psi(P, q)\left(\not p_{2}+m_{2}\right) \\
& =\int \frac{d^{3} \hat{q}^{\prime}}{(2 \pi)^{3}} K\left(\hat{q}, \hat{q}^{\prime}\right) \psi\left(\hat{q}^{\prime}\right),
\end{aligned}
$$

and the $4 \mathrm{D}$ BS wave function can be written as

$$
\Psi(P, q)=S_{F}\left(p_{1}\right) \Gamma(\widehat{q}) S_{F}\left(-p_{2}\right),
$$

where

$$
\Gamma(\widehat{q})=\int \frac{d^{3} \hat{q}^{\prime}}{(2 \pi)^{3}} K\left(\widehat{q}, \hat{q}^{\prime}\right) \psi\left(\hat{q}^{\prime}\right)
$$

plays the role of hadron-quark vertex function. Following a sequence of steps given in $[20,21]$, we obtain four Salpeter equations:

$$
\begin{aligned}
(M-2 \omega) \psi^{++}(\widehat{q}) & =-\Lambda_{1}^{+}(\widehat{q}) \Gamma(\widehat{q}) \Lambda_{2}^{+}(\widehat{q}), \\
(M+2 \omega) \psi^{--}(\widehat{q}) & =\Lambda_{1}^{-}(\widehat{q}) \Gamma(\widehat{q}) \Lambda_{2}^{-}(\widehat{q}), \\
\psi^{+-}(\widehat{q}) & =\psi^{-+}(\widehat{q})=0
\end{aligned}
$$

with the energy projection operators, $\Lambda_{j}^{ \pm}(\widehat{q})=\left(1 / 2 \omega_{j}\right)\left[\not P \omega_{j} /\right.$ $\left.M \pm I(j)\left(m_{j}+\not \hat{q}\right)\right], \omega_{j}^{2}=m_{j}^{2}+\hat{q}^{2}$, and $I(j)=(-1)^{j+1}$, where $j=1,2$ for quarks and antiquarks, respectively. The projected wave functions, $\psi^{ \pm \pm}(\widehat{q})$, in Salpeter equations are obtained by the operation of the above projection operators on $\psi(\hat{q})$ (for details see $[20,21])$ as

$$
\psi^{ \pm \pm}(\widehat{q})=\Lambda_{1}^{ \pm}(\widehat{q}) \frac{\not p}{M} \psi(\widehat{q}) \frac{\not p}{M} \Lambda_{2}^{ \pm}(\widehat{q}) .
$$

To obtain the mass spectral equation, we have to start with the above four Salpeter equations and solve the instantaneous Bethe-Salpeter equation. However, the last two equations do not contain eigenvalue $M$ and are thus employed to obtain constraint conditions on the Bethe-Salpeter amplitudes associated with various Dirac structures in $\psi(\widehat{q})$, as shown in detail in [21]. The framework is quite general so far. In fact the above four equations constitute an eigenvalue problem that should lead to evaluation of mass spectra of pseudoscalar and vector charmonium and bottomonium states such as $\eta_{c}$, 


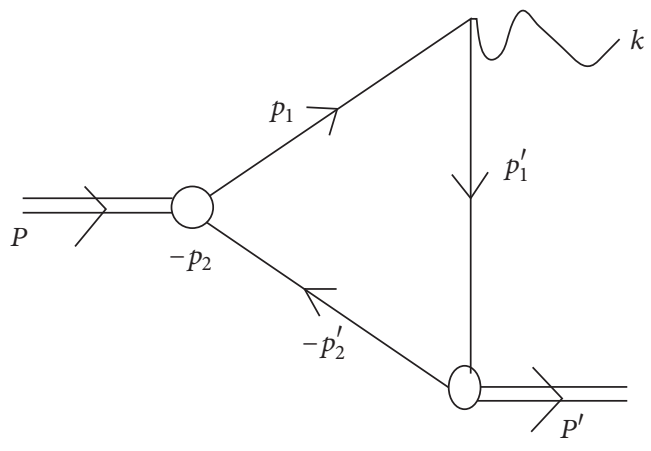

(a)

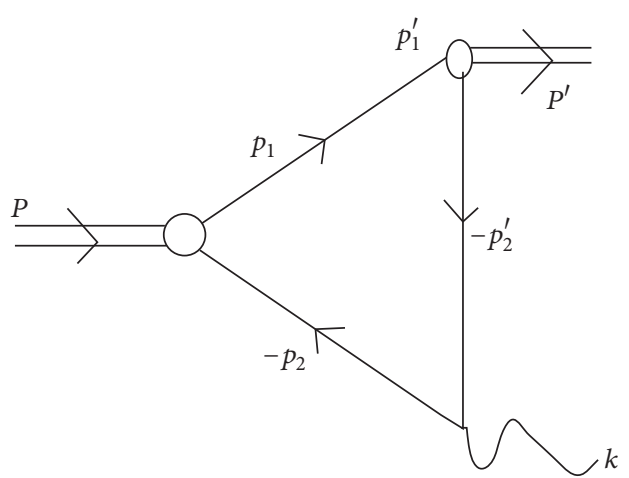

(b)

FIGURE 1: Feynman diagram for the first and second figures corresponding to the quark and antiquark emitting the photon, respectively, for the transition $V \rightarrow P \gamma$.

$\eta_{b}, J / \psi$, and $\Upsilon$ (see [21]). The numerical results [21] of mass spectra of $P$ and $V$ quarkonia participating in the radiative decays studied in this paper are listed in Section 3. We now give details of calculation of decay widths for the process, $V \rightarrow P+\gamma$, in the next section.

\section{Electromagnetic Transition of $V \rightarrow P+\gamma$}

The lowest order, Feynman diagrams for the process, $V \rightarrow$ $P \gamma$, are given in Figure 1, where $V\left(1^{--}\right)$and $P\left(0^{-+}\right)$are vector and pseudoscalar quarkonia, respectively. The second diagram is obtained from the first one by reversing the directions of internal fermionic lines.

The electromagnetic transition amplitude $M_{f i}$, corresponding to Figures 1(a) and 1(b), is given by the one-loop momentum integral as in $[23,31,32]$ :

$$
\begin{aligned}
& M_{f i}=-i e_{q} \int \frac{d^{4} q}{(2 \pi)^{4}} \\
& \cdot \operatorname{Tr}\left[\bar{\Psi}_{P}\left(P^{\prime}, q^{\prime}\right) \notin \Psi_{V}(P, q) S_{F}^{-1}\left(-p_{2}\right)\right. \\
& \left.\quad+\bar{\Psi}_{P}\left(P^{\prime}, q^{\prime}\right) S_{F}^{-1}\left(p_{1}\right) \Psi_{V}(P, q) \notin\right],
\end{aligned}
$$

where $P$ and $P^{\prime}$ are momenta of the initial and final hadrons, respectively, $p_{1}, p_{1}^{\prime},-p_{2}$, and $-p_{2}^{\prime}$ are initial and final momenta of the quark and antiquark, respectively, $k$ is the momentum of the outgoing photon, and $\Psi_{P}$ and $\Psi_{V}$ are the $4 \mathrm{D}$ BS wave functions of pseudoscalar and vector quarkonia involved in the process and are expressed as $\Psi_{V}(P, q)=$ $S_{F}\left(p_{1}\right) \Gamma(\widehat{q}) S_{F}\left(-p_{2}\right)$ and $\bar{\Psi}_{P}\left(P^{\prime}, q^{\prime}\right)=S_{F}\left(-p_{2}^{\prime}\right) \Gamma\left(\widehat{q^{\prime}}\right) S_{F}\left(p_{1}^{\prime}\right) \cdot e_{q}$ is the electric charge of quarks. $\epsilon_{\mu}$ is the polarization vector of the emitted photon. We have used the momentum relations $P=p_{1}+p_{2}, P^{\prime}=p_{1}^{\prime}+p_{2}^{\prime}, k=P-P^{\prime}$, and $p_{2}=p_{2}^{\prime}$ for first diagram. However for second diagram, the last momentum relation is $p_{1}=p_{1}^{\prime}$, while the other relations are the same as in first diagram. Here, the second term (coming from
Figure 1(b)) in (9) gives the same contribution as the first term (coming from Figure 1(a)), and hence we can write $M_{f i}$ as

$$
\begin{aligned}
& M_{f i} \\
& =-2 i e_{q} \int \frac{d^{4} q}{(2 \pi)^{4}} \operatorname{Tr}\left[\bar{\Psi}_{P}\left(P^{\prime}, q^{\prime}\right) \notin \Psi_{V}(P, q) S_{F}^{-1}\left(-p_{2}\right)\right]
\end{aligned}
$$

with an overall multiplicative factor of 2 .

This equation can be reduced to 3D forms by defining the $3 \mathrm{D}$ wave function, $\psi(\widehat{q})=(i / 2 \pi) \int M d \sigma \Psi(P, q)$. Thus, one can write the instantaneous Bethe-Salpeter form for the electromagnetic transition amplitude between two bound states as (see [33])

$$
\begin{aligned}
M_{f i} & =-\frac{2 e_{q}}{M} \int \frac{d^{3} \hat{q}}{(2 \pi)^{3}} \\
\cdot & \operatorname{Tr}\left\{\not p \bar{\psi}_{P}^{++}\left(\widehat{q}^{\prime}\right) \notin \psi_{V}^{++}(\widehat{q})-\not \supset \bar{\psi}_{P}^{--}\left(\hat{q}^{\prime}\right) \notin \psi_{V}^{--}(\widehat{q})\right\},
\end{aligned}
$$

where we resolve the amplitude corresponding to the first term (i.e., Figure 1(a)) in the previous equation into ++ and -- components, where the dominant contribution comes from the ++ components, while the contribution from the -- components is less than 1 percent [34]. Thus we can, to a good approximation, write the total amplitude for the process $V \rightarrow P \gamma$ in terms of only the ++ components as

$$
M_{f i}=-\frac{2 e_{q}}{M} \int \frac{d^{3} \widehat{q}}{(2 \pi)^{3}} \operatorname{Tr}\left\{\not p \bar{\psi}_{P}^{++}\left(\widehat{q}^{\prime}\right) \notin \psi_{V}^{++}(\widehat{q})\right\},
$$

where $\hat{q}^{\prime}=\widehat{q}+(1 / 2) \widehat{P}^{\prime}$ and $M$ is the mass of the initial quarkonia. The relativistic BS wave function of $P\left(0^{-+}\right)$meson bound state is taken as in [21]:

$$
\psi_{P}\left(\hat{q}^{\prime}\right)=N_{P}^{\prime}\left[M^{\prime}+P^{\prime}+\frac{\hat{q}^{\prime} P^{\prime}}{m}\right] \gamma_{5} \phi_{P}\left(\hat{q}^{\prime}\right),
$$

where $P^{\prime}$ is the momentum of the final state $P$-quarkonia, $N_{P}^{\prime}$ is the BS normalization of the final state $P$-quarkonia, and $M^{\prime}$ is the mass of the final state $P$-quarkonia. 
The relativistic BS wave function of $V\left(1^{--}\right)$quarkonium can ultimately be written as in [21]:

$$
\begin{aligned}
& \psi_{V}(\widehat{q}) \\
& =N_{V}\left[M \not \dot{q}+\hat{q} \cdot \varepsilon \frac{M}{m}+\not \not p+\frac{\not p \widehat{q} \cdot \varepsilon}{m}-\frac{\not p \phi \not d}{m}\right] \phi_{V}(\widehat{q}) .
\end{aligned}
$$

Here, $\varepsilon$ is the polarization vector of the vector quarkonia and $N_{V}$ is the BS normalizer of the initial state quarkonia. We wish to mention that (13) above is obtained by starting from the most general expression for $\Psi(P, q)$ in Eq. (17) of [21]. The general decomposition of instantaneous wave function in the center of mass frame of dimensionality $M$ is given in Eq. (18) of [21]. This instantaneous wave function $\Psi^{P}(\widehat{q})$ is put into the last two Salpeter equations, and the constraint conditions on the amplitudes (given in Eq. (19) of [21]) are obtained. We thus obtain Eq. (20) of [21]. This is then put into the first two Salpeter equations to obtain the two coupled equations (Eq. (21) of [21]). Solving them simultaneously leads to (13) of present paper (which is Eq. (23) in [21]), written in terms of $\phi_{P}$. Similarly we obtain $\Psi_{V}(\widehat{q})$ in (14) written in terms of $\phi_{V}$. These scalar functions $\phi_{P, V}(\widehat{q})$ satisfy the harmonic oscillator equation, Eq. (37) of [21], whose solutions are worked out by using the power series method in Eq. (37)-(41) of [21], with plots of these wave functions given in Figures 12 (for $P$-quarkonia) and Figures 3-4 (for $V$ quarkonia) in [21]. Thus we want to mention that the complete 2-fermion wave functions, $\Psi_{P, V}(\widehat{q})$, are ultimately expressed in terms of summation over various Dirac structures multiplying a single scalar function $\phi_{P, V}(\widehat{q})$, whose detailed algebraic expressions (Gaussian functions) are not approximations, but are obtained as analytic solutions of the algebraic form of the mass spectral equations for $P$ and $V$ quarkonia (with the complete spectrum written down in terms of principal quantum number $N$ in Eq. (35)-(36) of [21]) in an approximate harmonic oscillator basis.

The projected wave function for positive energy, $\psi^{++}(\widehat{q})$, is obtained by the operation of projection operators on $\psi_{P}\left(\left(^{\prime}\right)\right.$ and $\psi_{V}(\widehat{q})$, respectively, as in (8):

$$
\begin{aligned}
& \psi_{P}^{++}\left(\widehat{q}^{\prime}\right)=\Lambda_{1}^{+}\left(\widehat{q}^{\prime}\right) \frac{p^{\prime}}{M^{\prime}} \psi_{P}\left(\widehat{q}^{\prime}\right) \frac{p^{\prime}}{M^{\prime}} \Lambda_{2}^{+}\left(\widehat{q}^{\prime}\right) \\
& \psi_{V}^{++}(\widehat{q})=\Lambda_{1}^{+}(\widehat{q}) \frac{\not p}{M} \psi_{V}(\widehat{q}) \frac{\not p}{M} \Lambda_{2}^{+}(\widehat{q}),
\end{aligned}
$$

where $\Lambda_{1,2}^{+}(\widehat{q})=(1 / 2 \omega)[(\not p / M) \omega \pm m \pm \not h], \Lambda_{1,2}^{+}\left(\hat{q}^{\prime}\right)=$ $\left(1 / 2 \omega^{\prime}\right)\left[\left(P^{\prime} / M^{\prime}\right) \omega^{\prime} \pm m \pm \not \not^{\prime}\right]$, are called projection operators and $\omega^{2}=m^{2}+\hat{q}^{2}$ and $\omega^{\prime 2}=m^{2}+\hat{q}^{\prime 2}$. The relativistic positive energy wave function of equal mass pseudoscalar quarkonia in the center of mass system can be expressed as

$$
\begin{gathered}
\psi_{P}^{++}\left(\widehat{q}^{\prime}\right)=\frac{N_{P}^{\prime} \phi_{P}\left(\widehat{q}^{\prime}\right)}{2} \gamma_{5}\left\{M^{\prime}\left[\frac{m^{2}}{\omega^{\prime 2}}+\frac{m}{\omega^{\prime}}-\frac{\widehat{q}^{\prime 2}}{m \omega^{\prime}}\right]\right. \\
\left.-\not P^{\prime}\left[\frac{m^{2}}{\omega^{\prime 2}}+\frac{m}{\omega^{\prime}}\right]+\widehat{q}^{\prime} P^{\prime}\left[\frac{m}{\omega^{\prime 2}}+\frac{1}{\omega^{\prime}}\right]\right\} .
\end{gathered}
$$

And the relativistic positive energy wave function of equal mass vector quarkonia in the center of mass system can be written as

$$
\begin{aligned}
& \psi_{V}^{++}(\widehat{q})=\frac{N_{V} \phi_{V}(\widehat{q})}{2}\left\{M\left[\frac{m^{2}}{\omega^{2}}-\frac{m}{\omega}+\frac{\hat{q}^{2}}{m \omega}\right] \not+M \hat{q}\right. \\
& \cdot \varepsilon\left[\frac{m}{\omega^{2}}-\frac{1}{\omega}\right]+\left[\frac{m^{2}}{\omega^{2}}-\frac{m}{\omega}\right] \not \not P+\widehat{q} \cdot \varepsilon\left[\frac{m}{\omega^{2}}-\frac{1}{\omega}\right] \not p \\
& \left.-\left[\frac{m}{\omega^{2}}-\frac{1}{\omega}\right] \not P \not \not \phi d+\frac{\widehat{q} \cdot \varepsilon}{m \omega} \not \not p P-\frac{M \hat{q} \cdot \varepsilon}{m \omega} \not d\right\} \text {. }
\end{aligned}
$$

The conjugate of $\psi_{P}^{++}\left(\hat{q}^{\prime}\right)$ is evaluated from $\bar{\psi}_{P}^{++}\left(\hat{q}^{\prime}\right)=$ $\gamma^{0}\left(\psi_{P}^{++}\right)^{+} \gamma^{0}$, after a sequence of steps is expressed as

$$
\begin{gathered}
\bar{\psi}_{P}^{++}\left(\hat{q}^{\prime}\right)=-\frac{N_{P}^{\prime} \phi_{P}\left(\hat{q}^{\prime}\right)}{2} \gamma_{5}\left\{M^{\prime}\left[\frac{m^{2}}{\omega^{\prime 2}}+\frac{m}{\omega^{\prime}}-\frac{\hat{q}^{2}}{m \omega^{\prime}}\right]\right. \\
\left.-\not P^{\prime}\left[\frac{m^{2}}{\omega^{\prime 2}}+\frac{m}{\omega^{\prime}}\right]+\not \not^{\prime} \hat{q}^{\prime}\left[\frac{m}{\omega^{\prime 2}}+\frac{1}{\omega^{\prime}}\right]\right\} .
\end{gathered}
$$

Now, if we evaluate the total transition amplitude using (12), (17), and (18), we get

$$
M_{f i}=-\frac{e_{q} N_{P}^{\prime} N_{V}}{2 M} \int \frac{d^{3} \widehat{q}}{(2 \pi)^{3}} \phi_{P}\left(\widehat{q}^{\prime}\right) \phi_{V}(\widehat{q})[\mathrm{TR}],
$$

where

$$
\begin{aligned}
& {[\mathrm{TR}]=\operatorname{Tr}\left\{-\gamma_{5} \not P P^{\prime}\left[\frac{m^{2}}{\omega^{\prime 2}}+\frac{m}{\omega^{\prime}}\right]\right.} \\
& \cdot\left[M\left[\frac{m^{2}}{\omega^{2}}-\frac{m}{\omega}+\frac{\hat{q}^{2}}{m \omega}\right] \notin \notin-\left[\frac{m}{\omega^{2}}-\frac{1}{\omega}\right] \notin \not \not \phi \not \phi\right.
\end{aligned}
$$

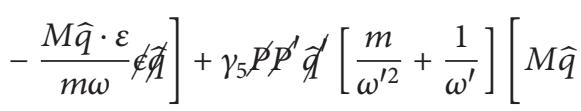

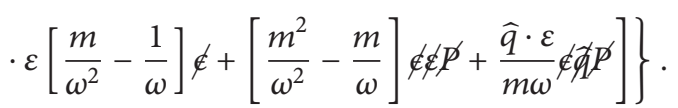

Evaluating trace over the gamma matrices, one can obtain the expression:

$$
\begin{aligned}
{[\mathrm{TR}]=} & -4 M \epsilon_{\mu \nu \alpha \beta} P_{\mu} P_{\nu}^{\prime} \epsilon_{\alpha} \varepsilon_{\beta}\left[\frac{m}{\omega^{\prime 2}}+\frac{1}{\omega^{\prime}}\right] \\
& \cdot\left[\frac{m^{3}}{\omega^{2}}-\frac{m^{2}}{\omega}+\left(\frac{m}{\omega^{2}}-\frac{1}{\omega}\right) \hat{q} \cdot \hat{q}^{\prime}\right],
\end{aligned}
$$

where $P$ and $P^{\prime}$ are momenta of the initial $(V)$ and final $(P)$ quarkonia, respectively. One can rewrite the transition amplitude after evaluation of the gamma matrices as

$$
\begin{aligned}
M_{f i}= & 2 e_{q} N_{P}^{\prime} N_{V} \int \frac{d^{3} \widehat{q}}{(2 \pi)^{3}} \phi_{P}\left(\widehat{q}^{\prime}\right) \phi_{V}(\widehat{q})\left[\frac{m}{\omega^{\prime 2}}+\frac{1}{\omega^{\prime}}\right] \\
& \cdot\left[\frac{m^{3}}{\omega^{2}}-\frac{m^{2}}{\omega}+\left(\frac{m}{\omega^{2}}-\frac{1}{\omega}\right) \widehat{q} \cdot \widehat{q}^{\prime}\right] \\
& \cdot \epsilon_{\mu \nu \alpha \beta} P_{\mu} P_{\nu}^{\prime} \epsilon_{\alpha} \varepsilon_{\beta} .
\end{aligned}
$$


The decay width for $V \rightarrow P \gamma$ can be expressed as (see [32])

$$
\begin{aligned}
\Gamma_{V \rightarrow P \gamma} & =\frac{\left|\vec{P}^{\prime}\right|}{8 \pi M^{2}}\left|M_{f i}\right|^{2} \\
\left|\vec{P}^{\prime}\right| & =\frac{1}{2 M}\left[M^{2}-M^{\prime 2}\right] .
\end{aligned}
$$

From (22) for total amplitude, $M_{\text {tot }}$, one can obtain

$$
\begin{aligned}
\left|M_{f i}\right|^{2} & =|f(\widehat{q})|^{2}\left(\epsilon_{\mu \nu \alpha \beta} P_{\mu} P_{\nu}^{\prime} \epsilon_{\alpha} \varepsilon_{\beta}\right)^{2} \\
& =\left(M^{4}+10 M^{2} M^{\prime 2}+M^{\prime 4}\right)|f(\widehat{q})|^{2},
\end{aligned}
$$

where

$$
\begin{aligned}
f(\widehat{q})= & 2 e_{q} N_{P}^{\prime} N_{V} \int \frac{d^{3} \hat{q}^{\prime}}{(2 \pi)^{3}} \phi_{P}\left(\hat{q}^{\prime}\right) \phi_{V}(\widehat{q})\left[\frac{m}{\omega^{\prime 2}}+\frac{1}{\omega^{\prime}}\right] \\
& \cdot\left[\frac{m^{3}}{\omega^{2}}-\frac{m^{2}}{\omega}+\left(\frac{m}{\omega^{2}}-\frac{1}{\omega}\right) \hat{q} \cdot \hat{q}^{\prime}\right] .
\end{aligned}
$$

One can obtain the expression of the decay rate of heavy quarkonia for the process, $V \rightarrow P \gamma$, as

$$
\begin{aligned}
& \Gamma_{V \rightarrow P \gamma} \\
& \quad=\frac{1}{64 \pi}\left[M^{3}+\frac{M^{\prime 4}}{M}-M^{\prime 2} M-\frac{M^{\prime 6}}{M^{3}}\right]^{3}|f(\widehat{q})|^{2} .
\end{aligned}
$$

$M^{\prime}$ and $M$ are the masses of the pseudoscalar heavy quarkonia $\left(\eta_{c}, \eta_{b}\right)$ and vector heavy quarkonia $(J / \psi, \Upsilon)$, respectively. In the expression for $f(\widehat{q}), N_{V}$ and $N_{P}^{\prime}$ are the BS normalizers for heavy vector and pseudoscalar quarkonia, respectively, which are given in a simple form as in [21]:

$$
\begin{aligned}
& N_{V}=\left[16 m M_{V} \int \frac{d^{3} \widehat{q}}{(2 \pi)^{3}} \frac{\hat{q}^{2}}{\omega^{3}} \phi_{V}^{2}(\widehat{q})\right]^{-1 / 2}, \\
& N_{P}^{\prime}=\left[\frac{16 M_{P}}{m} \int \frac{d^{3} \widehat{q}^{\prime}}{(2 \pi)^{3}} \frac{\hat{q}^{\prime 2}}{\omega^{\prime}} \phi_{P}^{2}\left(\widehat{q}^{\prime}\right)\right]^{-1 / 2} .
\end{aligned}
$$

The ground state $(1 S)$ wave function and the radial wave functions for the $2 S$ and $3 S$ excitations for initial heavy vector quarkonia are written as in Eq. (41) of [21]:

$$
\begin{aligned}
& \phi_{V}(1 S, \widehat{q})=\frac{1}{\pi^{3 / 4} \beta_{V}^{3 / 2}} e^{-\hat{q}^{2} / 2 \beta_{V}^{2}}, \\
& \phi_{V}(2 S, \widehat{q})=\left(\frac{3}{2}\right)^{1 / 2} \frac{1}{\pi^{3 / 4} \beta_{V}^{3 / 2}}\left(1-\frac{2 \widehat{q}^{2}}{3 \beta_{V}^{2}}\right) e^{-\hat{q}^{2} / 2 \beta_{V}^{2}}, \\
& \phi_{V}(3 S, \widehat{q}) \\
& \quad=\left(\frac{15}{8}\right)^{1 / 2} \frac{1}{\pi^{3 / 4} \beta_{V}^{3 / 2}}\left(1-\frac{20 \hat{q}^{2}}{15 \beta_{V}^{2}}+\frac{4 \widehat{q}^{4}}{15 \beta_{V}^{4}}\right) e^{-\hat{q}^{2} / 2 \beta_{V}^{2}}
\end{aligned}
$$

and the ground state $(1 S)$ wave function and the radial wave functions for the $2 S$ and $3 S$ excitations for final heavy pseudoscalar quarkonia are written as in Eq. (41) of [21]:

$$
\begin{aligned}
& \phi_{P}\left(1 S, \hat{q}^{\prime}\right)=\frac{1}{\pi^{3 / 4} \beta_{P}^{3 / 2}} e^{-\hat{q}^{\prime 2} / 2 \beta_{P}^{2}}, \\
& \phi_{P}\left(2 S, \hat{q}^{\prime}\right)=\left(\frac{3}{2}\right)^{1 / 2} \frac{1}{\pi^{3 / 4} \beta_{P}^{3 / 2}}\left(1-\frac{2 \widehat{q}^{\prime 2}}{3 \beta_{P}^{2}}\right) e^{-\widehat{q}^{\prime 2} / 2 \beta_{P}^{2}}, \\
& \phi_{P}\left(3 S, \hat{q}^{\prime}\right) \\
& \quad=\left(\frac{15}{8}\right)^{1 / 2} \frac{1}{\pi^{3 / 4} \beta_{P}^{3 / 2}}\left(1-\frac{20 \hat{q}^{\prime 2}}{15 \beta_{P}^{2}}+\frac{4 \hat{q}^{14}}{15 \beta_{P}^{4}}\right) e^{-\hat{q}^{\prime 2} / 2 \beta_{P}^{2}},
\end{aligned}
$$

where $\widehat{q}^{\prime}=\widehat{q}+(1 / 2)\left|\vec{P}^{\prime}\right|$ and $\left|\vec{P}^{\prime}\right|=\left(M_{V} / 2\right)\left(1-M_{P}^{2} / M_{V}^{2}\right)$.

The inverse range parameters for pseudoscalar and vector meson, respectively, are $\beta_{P}=$ $\left(4\left(m \omega_{q \bar{q}}^{2} / \sqrt{1+2 A_{0}(N+3 / 2)}\right)\right)^{1 / 4}$ and $\beta_{V}=$ $\left(2\left(m \omega_{q \bar{q}}^{2} / \sqrt{1+2 A_{0}(N+3 / 2)}\right)\right)^{1 / 4}$ and are dependent on the input kernel and contain the dynamical information, and they differ from each other only due to spin-spin interactions.

We had recently calculated the mass spectrum of ground and excited states of $P$ and $V$ quarkonia in [21]. The input parameters employed in this calculation that were fit from the mass spectrum of ground state pseudoscalar and vector quarkonia in [21] are given in Table 1.

The numerical values of inverse range parameters $\beta_{P}$ and $\beta_{V}$ for various $P$ and $V$ quarkonia in the radiative transitions studied in this paper are listed in Table 2 .

We had fixed the input parameters by studying the mass spectra for $P$ and $V$ quarkonia for a number of states. However in Tables 3 and 4, we list only the spectra of the quarkonia for which we have done calculations of radiative decay widths in this paper.

Numerical Results. We use the same input parameters listed in Table 1, to calculate the decay widths for the process $V \rightarrow$ $P+\gamma$. The results of radiative decay widths of our model are listed in Table 5. 
TABLE 3: Masses of ground and radially excited states of $\eta_{c}$ and $\eta_{b}$ (in GeV) in present calculation (BSE-CIA) along with experimental data and their masses in other models.

\begin{tabular}{lcccccc}
\hline & BSE - CIA & Expt. [4] & Pot. model [24] & QCD sum rule [7] & LQCD [25] & Re. P. model [26] \\
\hline$M_{\eta_{c}(1 S)}$ & 2.9509 & $2.983 \pm 0.0007$ & 2.980 & $3.11 \pm 0.52$ & 3.292 & 2.981 \\
$M_{\eta_{c}(2 S)}$ & 3.7352 & $3.639 \pm 0.0013$ & 3.600 & & 4.240 & 3.635 \\
$M_{\eta_{c}(3 S)}$ & 4.4486 & & 4.060 & & 3.989 \\
$M_{\eta_{b}(1 S)}$ & 9.0005 & $9.398 \pm 0.0032$ & 9.390 & $9.66 \pm 1.65$ & 7.377 & 9.398 \\
$M_{\eta_{b}(2 S)}$ & 9.7215 & $9.999 \pm 0.0028$ & 9.947 & & 8.202 & 9.990 \\
$M_{\eta_{b}(3 S)}$ & 10.4201 & & 10.291 & & & 10.329 \\
\hline
\end{tabular}

TABLE 4: Masses of ground and radially excited states of heavy vector quarkonium, $J / \psi$ and $\Upsilon$, in BSE-CIA along with their masses in other models and experimental data (all units are $\mathrm{GeV}$ ).

\begin{tabular}{lcccccc}
\hline & BSE - CIA & Expt. [4] & Rel. Pot. model [26] & Pot. model [24] & BSE [27] & LQCD [28] \\
\hline$M_{J / \psi(1 S)}$ & 3.0974 & $3.0969 \pm 0.000011$ & 3.096 & 3.0969 & 3.0969 & 3.099 \\
$M_{\psi(2 S)}$ & 3.6676 & $3.6861 \pm 0.00034$ & 3.685 & 3.6890 & 3.686 \\
$M_{\psi(3 S)}$ & 4.1945 & $4.03 \pm 0.001$ & 4.039 & 4.1407 & 4.065 \\
$M_{\Upsilon(1 S)}$ & 9.6719 & $9.4603 \pm 0.00026$ & 9.460 & 9.4603 & 9.460 & 4.099 \\
$M_{\Upsilon(2 S)}$ & 10.1926 & $10.0233 \pm 0.00031$ & 10.023 & 9.9814 & 10.029 \\
$M_{\Upsilon(3 S)}$ & 10.6979 & $10.3552 \pm 0.00005$ & 10.355 & 10.3195 & 10.379 \\
\hline
\end{tabular}

TABLE 5: Radiative decay widths of equal mass heavy vector quarkonium of ground state $(1 S)$ and radially excited states (2S, $3 S)$ in present calculation (BSE-CIA) along with their decay widths in other models and experimental data (all values are in unit of Kev).

\begin{tabular}{lccc}
\hline Transition & Our work & Expt. [4] & LFM [29] \\
\hline$\Gamma_{J / \psi(1 S) \rightarrow \eta_{c}(1 S) \gamma}$ & 2.0054 & $1.5687 \pm 0.011$ & $1.67 \pm 0.05$ \\
$\Gamma_{\psi(2 S) \rightarrow \eta_{c}(2 S) \gamma}$ & 0.5709 & $0.2093 \pm 0.002$ & \\
$\Gamma_{\psi(3 S) \rightarrow \eta_{c}(3 S) \gamma}$ & 0.2984 & & 0.4 \\
$\Gamma_{\Upsilon(1 S) \rightarrow \eta_{b}(1 S) \gamma}$ & 0.3387 & & $0.043_{-0.03}^{+0.09}$ \\
$\Gamma_{Y(2 S) \rightarrow \eta_{b}(2 S) \gamma}$ & 0.1053 & & 0.001 \\
$\Gamma_{Y(3 S) \rightarrow \eta_{b}(3 S) \gamma}$ & 0.0781 & & 0.0002 \\
\hline
\end{tabular}

\section{Discussions and Conclusion}

We have employed a $3 \mathrm{D}$ reduction of $\mathrm{BSE}$ (with a $4 \times 4$ representation for two-body $(q \bar{q})$ BS amplitude) under covariant instantaneous ansatz (CIA) and used it for calculating the amplitudes and decay widths for ground and radially excited states of vector $(J / \Psi$ and $\Upsilon)$ quarkonia in the process, $V \rightarrow$ $P+\gamma$.

The numerical values of decay widths calculated in this BSE framework for $(1 S, 2 S, 3 S)$ states of $J / \psi$ and $\Upsilon$ are shown in Table 5. The numerical calculation in this work has been done using Mathematica. We first fit our parameters to the ground state masses of $\eta_{c}, \eta_{b}, J / \psi$, and $\Upsilon$. Using the input parameters (along with the input quark masses) listed in Table 1, we obtained the best fit to these ground state masses. The same set of parameters above was used to calculate the masses of all the other (excited) states of $\eta_{c}, \eta_{b}, J / \psi$, and $\Upsilon$, as well as the leptonic decay constants of these states. Twophoton as well as two-gluon decay widths of $\eta_{c}$ and $\eta_{b}$ were further studied (see our recent paper [21]).

The results obtained for decay width of ground and radially excited states of $J / \psi$ and $\Upsilon$ are somewhat on the higher side in comparison to central values of data for these states, which might be probably due to the absence of the negative energy part employed in (11), where we have considered only the positive energy part (as in [34] employed earlier for heavy mesons). However, from Table 5, a wide range of variation of radiative decay widths of $J / \Psi(1 S)$ and $\Psi(2 S)$ states in different models can be observed. However, the important thing is that our radiative decay width values for vector quarkonia show a marked decrease as one goes from $1 S$ to $3 S$ state for $J / \psi$ and $\Upsilon$, which is again in conformity with data and other models. We have also given our predictions for radiative decay widths of $\Psi(3 S), \Upsilon(1 S), \Upsilon(2 S)$, and $\Upsilon(3 S)$ states, for which data is currently not yet available. The aim of doing this study was to mainly test our analytic forms of wave functions in (28)(29) obtained as solutions of mass spectral equations in an approximate harmonic oscillator basis obtained analytically from the $4 \times 4 \mathrm{BSE}$ as our starting point (that had so far given good predictions for leptonic decays of $P$ and $V$ quarkonia and the two-photon and the two-gluon decays of $P$-quarkonia [21]), to the single photon radiative decays of $V$ quarkonia. This would in turn lead to validation of our approach, which provides a much deeper insight than the purely numerical calculations in $4 \times 4 \mathrm{BSE}$ approach that are prevalent in the literature. 
In the process of arriving at analytic solution of spectra by solving the coupled Salpeter equations, we have worked in approximate harmonic oscillator basis, also employing approximation $\omega \sim m$ for heavy quarks. We do concede that some numerical accuracy has been lost in the process, but at the same time we have obtained a much deeper understanding of the mass spectra of quarkonia, where the equations are expressible in terms of the principal quantum number $N$. We wish to mention that to the best of our knowledge we have not encountered any work in $4 \times 4$ representation of BSE that treats this problem analytically. On the contrary all the other $4 \times 4$ approaches adopt a purely numerical approach of solving the coupled set of Salpeter equations, which may enhance the numerical accuracy, but this is at the expense of a deeper understanding of the spectral problem. We further wish to mention that the correctness of our approximations can be judged by the fact that our plots of wave functions obtained analytically for various states of $P$ and $V$ quarkonia [21] are very similar to the corresponding plots of wave functions of various states of these quarkonia obtained by purely numerical approach in [13].

We are also not aware of any other BSE framework, involving $4 \times 4 \mathrm{BS}$ amplitude, with all the Dirac structures incorporated (in fact many works use only the leading Dirac structure), that treats these problems analytically and uses the algebraic forms of wave functions derived analytically from mass spectral equation for calculation of various transitions. To the best of our knowledge, all the other $4 \times 4$ BSE approaches treat this problem numerically after obtaining the coupled set of equations.

\section{Conflicts of Interest}

The authors declare that they have no conflicts of interest.

\section{References}

[1] B. Aubert, M. Bona, Y. Karyotakis et al., "Evidence for the $\eta_{b}(1 S)$ Meson in Radiative $\Upsilon(2 s)$ Decay," Physical Review Letters, vol. 103, Article ID 161801, 2009, (BaBar collaboration).

[2] K.-F. Chen, W.-S. Hou, I. Adachi et al., "Observation of an enhancement in $\mathrm{e}^{+} \mathrm{e}^{-} \rightarrow \Upsilon(1 S) \pi^{+} \pi^{-}, \Upsilon(2 S) \pi^{+} \pi^{-}$, and $\Upsilon(3 S) \pi^{+} \pi^{-}$production near $\sqrt{ } \mathrm{s}=10.89 \mathrm{GeV}$," Physical Review $D$ - APS Physics, vol. 82, (Belle Collaboration), Article ID 091106, 2010.

[3] K. W. Edwards, R. Janicek, P. M. Patel et al., "Study of $B$ Decays to Charmonium States: $B \rightarrow \eta_{c} \mathrm{~K}$ and $B \rightarrow \chi_{c 0} \mathrm{~K}$," Physical Review Letters, vol. 86, no. 30, 2001, (CLEO collaboration).

[4] K. Olive, K. Agashe, and C. Amsler, "Review of particle physics," Chinese Physics C, vol. 38, no. 9, Article ID 090001, 2014, (Particle Data Group).

[5] C. McNielle, C. T. H. Davies, E. Follana, K. Hornbostel, and G. P. Lepage, "Heavy meson masses and decay constants from relativistic heavy quarks in full lattice QCD," Physical Review D, vol. 86, 16 pages, 2012, (HPQCD Collaboration).

[6] J. Gasser and H. Leutwyler, "Chiral perturbation theory to one loop," Annals of Physics, vol. 158, no. 1, pp. 142-210, 1984.

[7] E. Veli Veliev, K. Azizi, H. Sundu, and N. Aksit, "Investigation of heavy-heavy pseudoscalar mesons in thermal QCD sum rules," Journal of Physics G, vol. 39, Article ID 015002, 2012.
[8] M. Neubert, "Heavy-quark symmetry," Physics Reports, vol. 245, 259 pages, 1994.

[9] G. T. Bodwin, E. Braaten, and G. P. Lepage, "Rigorous QCD analysis of inclusive annihilation and production of heavy quarkonium," Physical Review D, vol. 51, article 1125, p. 1125, 1995.

[10] C. H. L. Smith, "A relativistic formulation of the quark model for mesons," Annals of Physics, vol. 53, 521 pages, 1969.

[11] A. N. Mitra and B. M. Sodermark, "A dynamical principle for 3D-4D interlinkage in Salpeter-like equations," Nuclear Physics A, vol. 695, no. 1-4, pp. 328-352, 2001.

[12] R. Alkofer and L. von Smekal, “The infrared behaviour of QCD Green's functions: confinement, dynamical symmetry breaking, and hadrons as relativistic bound states," Physics Reports A: Review Section of Physics Letters, vol. 353, no. 5-6, pp. 281-465, 2001.

[13] C.-H. Chang and G. L. Wang, "Spectrum for heavy quarkonia and mixture of the relevant wave functions within the framework of Bethe-Salpeter equation," Science in China G, vol. 53, 2010, G53.

[14] R. Ricken, M. Koll, D. Merten, B. Metsch, and H. Petry, "A relativistic quark model for mesons with an instanton-induced interaction," The European Physical Journal A, vol. 9, p. 221, 2000.

[15] A. N. Mitra and S. Bhatnagar, "A hadron-quark vertex function: interconnection between $3 \mathrm{D}$ and $4 \mathrm{D}$ wave functions," International Journal of Modern Physics A, vol. 7, p. 121, 1992.

[16] S. Patel, P. C. Vinodkumar, and S. Bhatnagar, "Decay rates of charmonia within a quark-antiquark confining potentials," Chinese Physics C, vol. 40, no. 5, Article ID 053102, 2016.

[17] A. N. Mitra, "QCD motivated BSE-SDE framework for quark dynamics under Markov-Yukawa transversality- a unified view of qq-bar and qqq systems," Proceedings of the Indian National Science Academy, vol. 65, p. 527, 1999.

[18] S. Bhatnagar, D. S. Kulshreshtha, and A. N. Mitra, "How big are the decay constants $f_{P}$ of heavy-light mesons?" Physics Letters $B$, vol. 263, no. 3-4, pp. 485-490, 1991.

[19] S. Bhatnagar, J. Mahecha, and Y. Mengesha, "Relevance of various Dirac covariants in hadronic Bethe-Salpeter wave functions in electromagnetic decays of ground state vector mesons," Physical Review D, vol. 90, Article ID 014034, 2014.

[20] H. Negash and S. Bhatnagar, "Mass spectrum and leptonic decay constants of ground and radially excited states of $\eta_{c}$ and $\eta_{b}$ in a Bethe-Salpeter equation framework," International Journal of Modern Physics E, vol. 24, no. 4, Article ID 1550030, 2015.

[21] H. Negash and S. Bhatnagar, "Spectroscopy of ground and excited states of pseudoscalar and vector charmonium and bottomonium," International Journal of Modern Physics E, vol. 25, Article ID 1650059, 29 pages, 2016.

[22] C.-H. Chang, J.-K. Chen, and G.-L. Wang, "Instantaneous formulation for transitions between two instantaneous bound states and its gauge invariance," Communications in Theoretical Physics, vol. 46, no. 3, pp. 467-480, 2006.

[23] C-H. Chang, J.-K. Chen, and G.-L. Wang, "The instantaneous formulations for bethe salpeter equation and radiative transitions between two bound-states," https://arxiv.org/abs/hep-th/ 0312250 .

[24] K. B. V. Bhagyesh, A. P. Kumar, and J. Monterio, "Heavy quarkonium spectra and its decays in a nonrelativistic model with Hulthen potential," Journal of Physics G: Nuclear and Particle Physics, vol. 38, no. 8, Article ID 085001, 2011. 
[25] T. Burch, C. DeTar, M. Di Pierro et al., "Quarkonium mass splittings in three-flavor lattice QCD," Physical Review D, vol. 81, Article ID 034508, 2010.

[26] D. Ebert, R. N. Faustov, and V. O. Galkin, "Spectroscopy and Regge trajectories of heavy quarkonia in the relativistic quark model," Physics of Atomic Nuclei, vol. 76, no. 12, pp. 1554-1562, 2013.

[27] G.-L. Wang, "Decay constants of heavy vector mesons in relativistic Bethe-Salpeter method," Physics Letters B, vol. 633, 2006.

[28] T. Kawanai and S. Sasaki, "Potential description of the charmonium from lattice QCD," AIP Conference Proceedings, September 2016.

[29] H.-M. Choi, "Decay constants and radiative decays of heavy mesons in light-front quark model," Physical Review D, Article ID 073016, 2007.

[30] S. F. Radford and W. W. Repko, "Potential model calculations and predictions for heavy quarkonium," Physical Review D Particles, Fields, Gravitation and Cosmology, vol. 75, no. 7, Article ID 074031, 2007.

[31] N. N. Singh and A. N. Mitra, "Hadronic transition amplitudes under null-plane Bethe-Salpeter dynamics," Physical Review D, vol. 38, no. 5, article 1454, pp. 1454-1468, 1988.

[32] E. Mengesha and S. Bhatnagar, "Radiative decays of equal mass vector mesons in a Bethe-Salpeter equation framework," International Journal of Modern Physics E, vol. 21, Article ID 1250084, 2012.

[33] G.-H. Wang and G.-L. Wang, "Radiative E1 decays of X(3872)," Physics Letters B, vol. 9, no. 4, 9 pages, 2011.

[34] T. Wang, G.-L. Wang, Y. Jiang, and W.-L. Ju, "EM Decay of $\mathrm{X}(3872)$ as the $1^{1} \mathrm{D}_{2}\left(2^{-+}\right)$charmonium," Journal of Physics $G$ : Nuclear and Particle Physics, vol. 2013, 2013. 

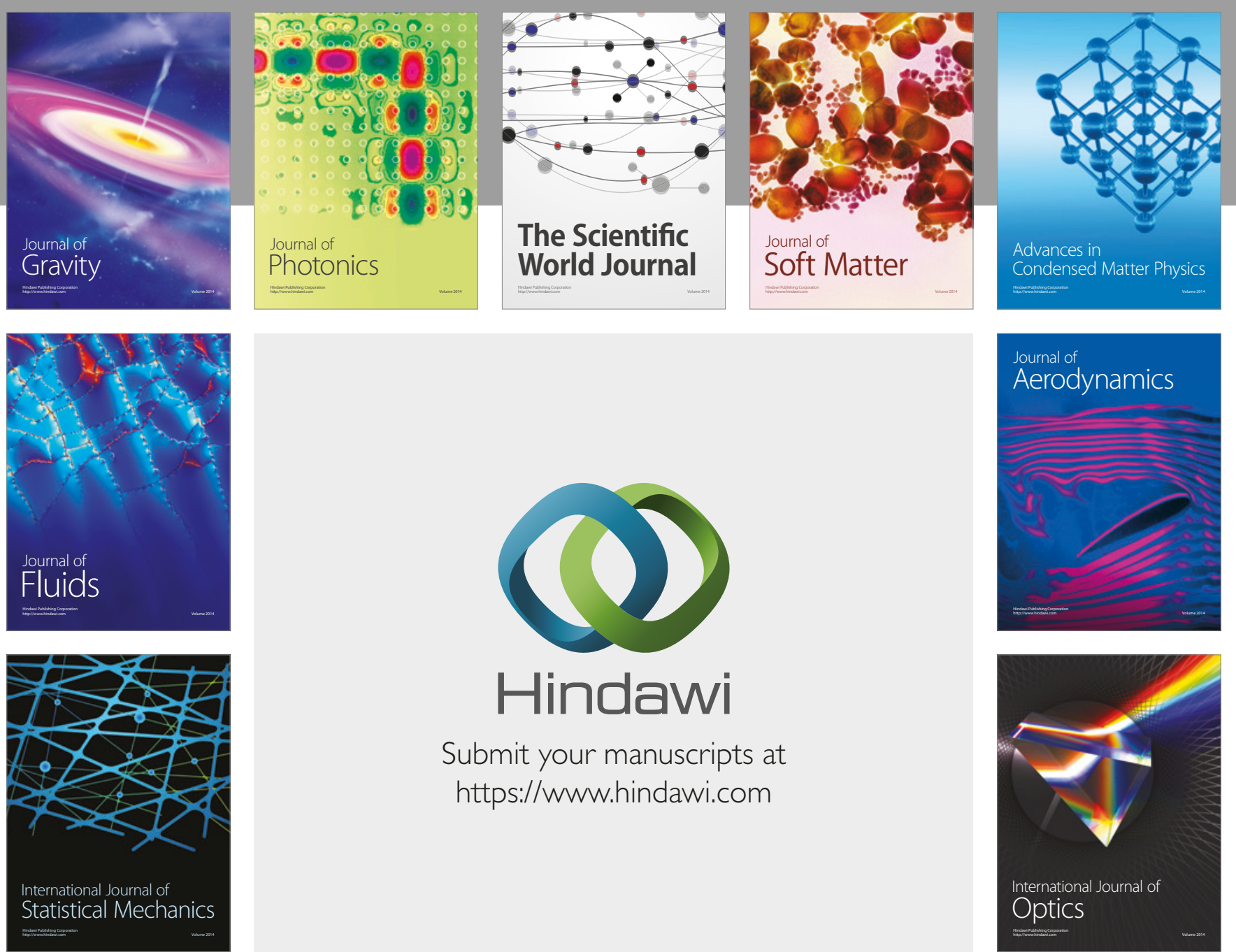

Submit your manuscripts at

https://www.hindawi.com
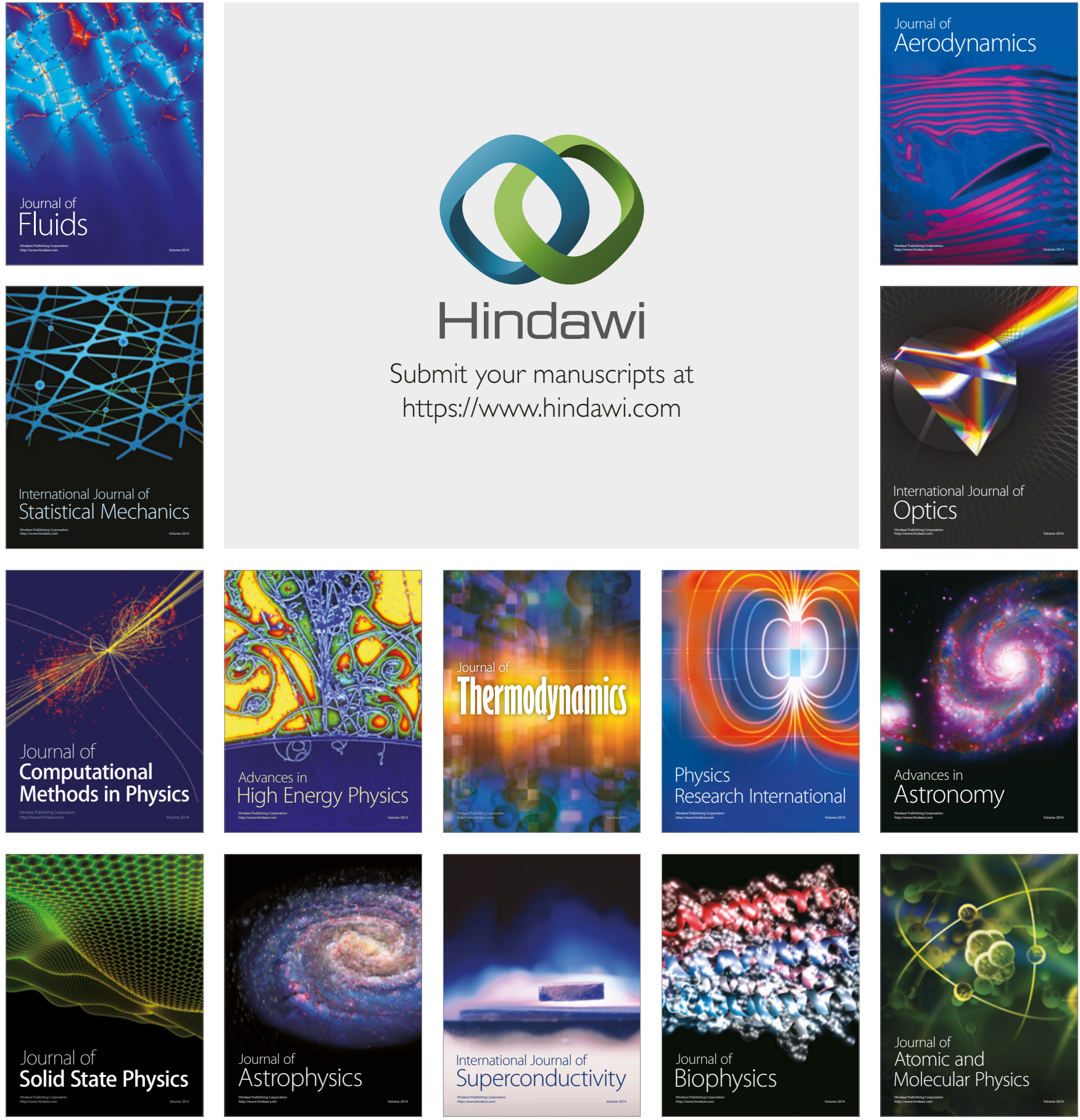